\title{
Hybrids of Thiazolidin-4-Ones and 1,3,4-Thiadiazole: Synthesis and Biological Screening of A Potential New Class of Acetylcholinesterae Inhibitors
}

\author{
Navidha Aggarwal 1,*俛, Sandeep Jain ${ }^{1}$, Neetu Chopra ${ }^{1(\mathbb{D})}$ \\ 1 Department of Pharmaceutical Sciences, Guru Jambheshwar University of Science and Technology, Hisar-125001, \\ Haryana, India \\ * Correspondence: navidhabansal16@gmail.com (N.A.);
}

Scopus Author ID 35725336400

Received: 20.04.2021; Revised: 5.06.2021; Accepted: 10.06.2021; Published: 8.08.2021

\begin{abstract}
Using propanoic acid and thiosemicarbazide as starting materials, a new sequence of thiazolidin-4-one analogs with thiadiazole derivative was synthesized in appreciable yield. Spectral techniques such as IR, ${ }^{1} \mathrm{H}$ NMR, ${ }^{13} \mathrm{C}$ NMR, and MS were used to validate the structures of the synthesized compounds (4a-t). In vitro acetylcholinesterase inhibitory activity of these synthesized compounds was assessed using an Ellman's method spectrophotometer and donepezil as a standard drug. Compounds 2-((5-ethyl-1,3,4-thiadiazol-2-yl)imino)-5-(4-methylbenzylidene)thiazolidin-4one(4o) and 5-(4-(benzyloxy)benzylidene)-2-((5-ethyl-1,3,4-thiadiazol-2-yl)imino)thiazolidin-4one(4i) were found to be potent $\mathrm{AChE}$ enzyme inhibitors, with pIC $\mathrm{C}_{50}(\mathrm{mM})$ values of $1.30 \pm 0.007$ and $1.22 \pm 0.002$, respectively. Finally, these significant results could pave the way for the development of new AChE inhibitors and will serve as the basis for future research.
\end{abstract}

Keywords: acetylcholinesterase inhibitors;thiadiazole; Ellman's method; thiazolidin-4-one;

Alzheimer's disease.

(C) 2021 by the authors. This article is an open-access article distributed under the terms and conditions of the Creative Commons Attribution (CC BY) license (https://creativecommons.org/licenses/by/4.0/).

\section{Introduction}

Alzheimer's disease (AD) is a neurodegenerative disease that causes memory loss and the inability to remember recent events or conversations. As the disease progresses, the patient develops extreme memory failure and loses the ability to perform daily activities [1,2]. Dementia affects 50 million people worldwide, with 30-35 million of those suffering from Alzheimer's disease [3]. Cortical cholinergic neurotransmission dysfunction is associated with Alzheimer's disease. The new Alzheimer's disease pharmacotherapy is focused on the cholinergic hypothesis, to increase acetylcholine (Ach) levels by inhibiting the enzyme acetylcholinesterase (AChE) [4].

As a result, the most successful therapies are those that aim to raise acetylcholine levels in the brain, an essential neurotransmitter whose levels drop as the disease progresses. AChE inhibitors function by preventing the enzyme AChE from breaking down acetylcholine. However, the adverse effects such as nausea, vomiting, bradycardia, and weight loss have limited the therapeutic effectiveness of AChE inhibitors. In this context, medicinal chemists have a complicated challenge in developing robust and novel drugs with better pharmacological profiles and therapeutic efficacy to manage Alzheimer's disease [5]. 
In pharmaceutical chemistry, nitrogen and sulfur-containing heterocycles, such as thiazolidinone and 1,3,4-thiadiazoles, are often used in drug design. These molecule scaffolds have biological activity that can be directed to various targets with the right modifications [6]. Structure modifications in thiazolidinone have resulted in acetylcholinesterase inhibitors [7], [8], butyrylcholinesterase and glucosidase inhibitors [7], aldose reductase inhibitors [9], alphaamylase and urease inhibitors [10], anti-cancer [11-13], antitrypanosomal [11], antimicrobial [14-20], COX-2 inhibitor [21], antifungal [22, 23], antimycobacterial [23], anti-inflammatory [24].

Another five-membered 1,3,4-thiadiazole ring has been reported to possess various pharma- cological activities, including acetylcholinesterase inhibitors [25], antibacterial [2628], antifungal [29,30], anti-cancer [31-33], antimicrobial [31,34,35], antioxidant [35], $\beta$ glucuronidase inhibitors [36], analgesic and anti-inflammatory [37, 38].

The combination of two bioactive moieties has emerged as a promising drug development technique. The hybridization of thiazolidinones with 1,3,4-thiadiazole to obtain single biologically active entities is defined in this paper. These newly synthesized derivatives were tested for their AChE inhibitory activity in the treatment of Alzheimer's disease

\section{Materials and Methods}

\subsection{Materials.}

In the experiment, all of the chemicals used were of analytical grade. Acetylcholinesterase enzyme obtained from Electrophorus electricus (C3389), 5,5'-Dithiobis-(2-nitrobenzoic acid ) (DTNB), Acetylthiocholine iodide (ATCI) were purchased from Sigma -Aldrich.

\subsection{Chemistry.}

Thin-layer chromatography (TLC) with silica gel G was used to monitor the progress of the reactions. The melting points (m.p.) were determined using a Decibel melting point apparatus that was not corrected. The potassium bromide pellet method was used to record infrared spectra (IR) on a Perkin Elmer IR spectrophotometer. The nuclear magnetic resonance spectra $\left({ }^{1} \mathrm{H}\right.$ NMR and ${ }^{13} \mathrm{C}$ NMR) were recorded on the Bruker Avance III $400 \mathrm{MHz}$ NMR spectrophotometer. Chemical shifts are expressed as $\delta$ values in parts per million (ppm) downfield from the internal standard tetramethylsilane (TMS). The LC-MS/MS QTOF MakeSCIEX mass spectrometer was used to record mass spectra. Synthesis of final derivatives (4(at)) outlined in Scheme 1.

\subsubsection{Synthesis of 2-amino-5-ethyl-1,3,4-thiadiazole (I).}

In a round bottom flask, a mixture of propanoic acid $(0.06 \mathrm{~mol})$ and thiosemicarbazide ( $0.072 \mathrm{~mol})$ in concentrated $\mathrm{H}_{2} \mathrm{SO}_{4}(90 \mathrm{ml})$ was refluxed for 10-13 hours on a water bath. The mixture was then cooled before being neutralized with an ammonia solution. Separate the solids, filter them, and wash them with distilled water. Ethanol was used to recrystallize the product [39]. 
2.2.2. Synthesis of 1-(5-ethyl-1,3,4-thiadiazol-2-yl)thiourea (II).

Intermediate-I ( $0.04 \mathrm{~mol})$ was added to a minimum amount of dilute $\mathrm{HCl}$, which was then treated with ammonium thiocyanate $(0.08 \mathrm{~mol})$. After that, the mixture was refluxed for 3-4 hours in a water bath. The mixture was then poured over ice-cold water. The separated substance was filtered and washed with distilled water. Ethanol was used to recrystallize the product [40].

2.2.3. Synthesis of 2-(5-ethyl-1,3,4-thiadiazol-2-ylimino)thiazolidin-4-one (III).

In a solution of intermediate-II $(0.02 \mathrm{~mol})$ in dimethylformamide (DMF), chloroacetic acid $(0.02 \mathrm{~mol})$ and sodium acetate $(0.02 \mathrm{~mol})$ were added and refluxed for $18-21$ hours. The mixture was then poured over crushed ice. The precipitate was filtered and washed with distilled water several times. Alcohol was used to recrystallize the product [41].

2.2.4. Synthesis of final derivatives (4a-t).

In glacial acetic acid, intermediate-III $(0.01 \mathrm{~mol})$ was mixed with anhydrous sodium acetate $(0.01 \mathrm{~mol})$ and aldehyde derivatives $(0.01 \mathrm{~mol})$. After that, the mixture was subjected to 4-5 hours of reflux. After that, the mixture was then allowed to cool. The separated product was filtered and washed with warm water. The filtered product (4a-t) was recrystallized with ethanol to obtain the final compounds [42].

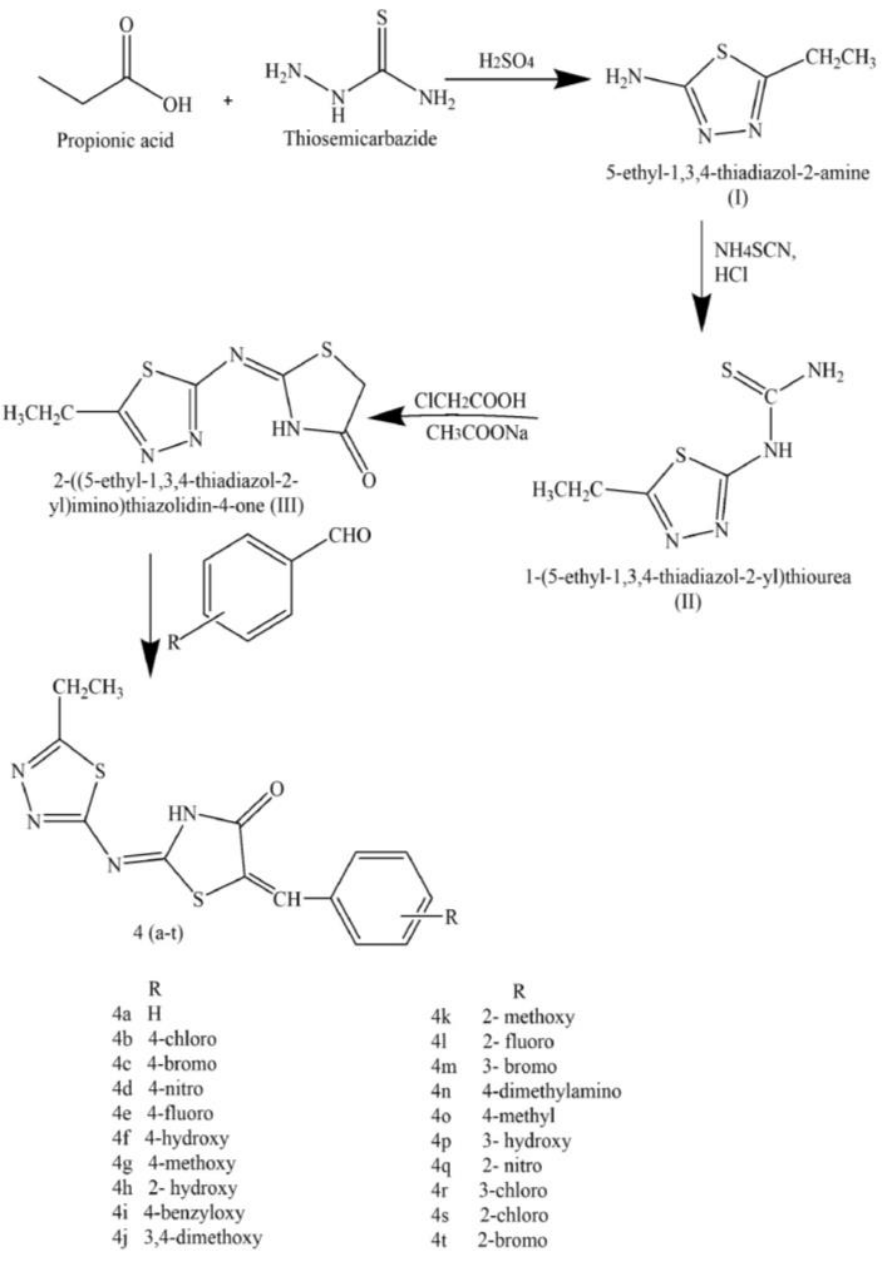

Scheme 1. Synthesis of final derivatives (4(a-t)). 


\section{5-(benzylidene)-2-((5-ethyl-1,3,4-thiadiazol-2-yl)imino)thiazolidin-4-}

one(4a):Yield: $83.67 \%$; m.p. $208-210^{\circ} \mathrm{C}$; IR ( $\mathrm{KBr}$ pellets) $\mathrm{cm}^{-1}: 1642.79(\mathrm{C}=\mathrm{N}$ str.), 1335.72 (C-N str.), 3275.68 (N-H str., thiazolidine ring), 1695.77 (C=O str., thiazolidin-4-one), 602.05 (C-S bend), 1595.71 (C=C str.), 3014 (C-H str., aliphatic), 3114 (C-H str., aromatic ring),; ${ }^{1} \mathrm{H}$ NMR (400 MHz, DMSO-d6): $\delta$ 6.98-7.82 (m, 5H, Ar-H), 12.24 (s, 1H, NH), $7.72(\mathrm{~s}, 1 \mathrm{H},-$ $\mathrm{CH}=$ ), 2.12 (q, $2 \mathrm{H}, \mathrm{CH}_{2}$ of thiadiazole), 1.12 (t, 3H, $\mathrm{CH}_{3}$ of thiadiazole); ${ }^{13} \mathrm{C}$ NMR (DMSO$\left.\mathrm{d}_{6}\right): \delta 169.4,159.6,152.6,140.4,132.6,122.4,121.6,120.4,22.2,15.4 ; \mathrm{MS}: \mathrm{m} / z\left(\mathrm{M}^{+}+1\right.$ 317.10).

\section{5-(4-chlorobenzylidene)-2-((5-ethyl-1,3,4-thiadiazol-2-yl)imino)thiazolidin-4-}

one(4b): Yield: $79.65 \%$; m.p. $240-242^{\circ} \mathrm{C}$; IR (KBr pellets) $\mathrm{cm}^{-1}: 1641.72$ (C=N str.), 1339.78 (C-N str.), 3277.06 (N-H str., thiazolidine ring), 1702 (C=O str., thiazolidin-4-one), 691.37 (C$\mathrm{S}$ bend), 1601.72 (C=C str.), 3042.01 (C-H str., aliphatic), 3117.32 (C-H str., aromatic ring), 752(C-Cl bend); ${ }^{1} \mathrm{H}$ NMR (400 MHz, DMSO-d 6 ): $\delta$ 6.96-7.82 (m, 4H, Ar-H), $12.45(\mathrm{~s}, 1 \mathrm{H}$, $\mathrm{NH}), 7.79(\mathrm{~s}, 1 \mathrm{H},-\mathrm{CH}=), 2.21$ (q, $2 \mathrm{H}, \mathrm{CH}_{2}$ of thiadiazole), 1.29 (t, $3 \mathrm{H}, \mathrm{CH}_{3}$ of thiadiazole); ${ }^{13} \mathrm{C}$ NMR (DMSO-d $)$ : $\delta 171.1,158.4,153.8,139.8,135.6,129.9,128.2,127.6,118.6,23.3,15.6$; MS: $m / z\left(\mathrm{M}^{+}+1\right.$ 351.92).

\section{5-(4-bromobenzylidene)-2-((5-ethyl-1,3,4-thiadiazol-2-yl)imino)thiazolidin-4-} one(4c): Yield: $84.28 \%$; m.p. $241-243^{\circ} \mathrm{C}$; IR ( $\mathrm{KBr}$ pellets) $\mathrm{cm}^{-1}$ : 1617.94 (C=N str.), 1375.12 (C-N str.), 3116.92 (N-H str., thiazolidine ring), 1610 (C=O str., thiazolidin-4-one), 620.65 (C$\mathrm{S}$ bend), 1627 (C=C str.), 3047.61 (C-H str., aliphatic), 2924.36 (C-H str., aromatic ring), 533.07 (C-Br bend); ${ }^{1} \mathrm{H}$ NMR (400 MHz, DMSO-d6): $\delta$ 7.21-7.92 (m, 4H, Ar-H), $12.21(\mathrm{~s}, 1 \mathrm{H}$, $\mathrm{NH}), 7.81(\mathrm{~s}, 1 \mathrm{H},-\mathrm{CH}=), 2.29$ (q, $2 \mathrm{H}, \mathrm{CH}_{2}$ of thiadiazole), $1.24\left(\mathrm{t}, 3 \mathrm{H}, \mathrm{CH}_{3}\right.$ of thiadiazole); ${ }^{13} \mathrm{C}$ NMR (DMSO-d6): $\delta 169.6,157.4,155.8,139.8,132.8,125.4,128.6,122.2,117.9,22.7,16.2$; MS: $m / z\left(\mathbf{M}^{+}+1396.02\right)$.

\section{2-((5-ethyl-1,3,4-thiadiazol-2-yl)imino)-5-(4-nitrobenzylidene)thiazolidin-4-}

one(4d):Yield: $72.57 \%$; m.p. 203-205 ${ }^{\circ} \mathrm{C}$; IR ( $\mathrm{KBr}$ pellets) $\mathrm{cm}^{-1}: 1602.61$ (C=N str.), 1384.24 (C-N str.), 3448.14 (N-H str., thiazolidine ring), 1799.30 (C=O str., thiazolidin-4-one), 645.96 (C-S bend), 1507.83 (C=C str.), 2860 (C-H str., aliphatic), 3114.37 (C-H str., aromatic ring), 1438.70 ( $\mathrm{NO}_{2}$ assym. str.), 1384.24 ( $\mathrm{NO}_{2}$ symm. Str.); ${ }^{1} \mathrm{H}$ NMR (400 MHz, DMSO-d6): $\delta$ 7.378.38 (m, 4H, Ar-H), 12.29 (s, 1H, NH), 7.92 (s, 1H, - $\mathrm{CH}=), 2.27$ (q, 2H, $\mathrm{CH}_{2}$ of thiadiazole), 1.26 (t, 3H, $\mathrm{CH}_{3}$ of thiadiazole); ${ }^{13} \mathrm{C}$ NMR (DMSO-d6): $\delta$ 169.6, 156.6, 154.3, 144.8, 139.6, 138.3, 130.8, 127.3, 115.4, 22.4, 14.8; MS: $m / z\left(\mathrm{M}^{+}+1362.22\right)$.

2-((5-ethyl-1,3,4-thiadiazol-2-yl)imino)-5-(4-fluorobenzylidene) thiazolidin-4-one (4e): Yield: $81.23 \%$; m.p. $250-252^{\circ} \mathrm{C}$; IR (KBr pellets) $\mathrm{cm}^{-1}: 1604.60$ (C=N str.), 1336.23 (C$\mathrm{N}$ str.), 3453.50 (N-H str., thiazolidine ring), 1702.99 (C=O str., thiazolidin-4-one), 609.77 (C$\mathrm{S}$ bend), 1508.60 ( $\mathrm{C}=\mathrm{C}$ str.), 3050 ( $\mathrm{C}-\mathrm{H}$ str., aliphatic), 3283.62 (C-H str., aromatic ring), 1227.23 (C-F bend); ${ }^{1} \mathrm{H}$ NMR (400 MHz, DMSO-d 6 ): $\delta$ 6.96-7.89 (m, 4H, Ar-H), $12.34(\mathrm{~s}, 1 \mathrm{H}$, $\mathrm{NH}), 7.82$ (s, $1 \mathrm{H},-\mathrm{CH}=), 2.13\left(\mathrm{q}, 2 \mathrm{H}, \mathrm{CH}_{2}\right.$ of thiadiazole), 1.21 (t, $3 \mathrm{H}, \mathrm{CH}_{3}$ of thiadiazole); ${ }^{13} \mathrm{C}$ NMR (DMSO-d6): $\delta$ 170.8, 155.6, 155.4, 154.6, 140.2, 125.4, 125.0, 116.8, 111.3, 22.6, 15.5; MS: $m / z\left(\mathrm{M}^{+}+1335.15\right)$.

\section{2-((5-ethyl-1,3,4-thiadiazol-2-yl)imino)-5-(4-hydroxybenzylidene)thiazolidin-4-}

one(4f): Yield: $76.57 \%$; m.p. $211-213^{\circ} \mathrm{C}$; IR (KBr pellets) $\mathrm{cm}^{-1}: 1608.39$ (C=N str.), 1341.33 (C-N str.), 3562 (N-H str., thiazolidine ring), 1776.22 (C=O str., thiazolidin-4-one), 617.99 (C$\mathrm{S}$ bend), 1596.16 (C=C str.), 3052 (C-H str., aliphatic), 3115 (C-H str., aromatic ring), 3422.19 (OH str.); ${ }^{1} \mathrm{H}$ NMR (400 MHz, DMSO-d 6 ): $\delta$ 6.92-7.79 (m, 4H, Ar-H), 12.40 (s, 1H, NH), 7.85 (s, $1 \mathrm{H},-\mathrm{CH}=), 2.26\left(\mathrm{q}, 2 \mathrm{H}, \mathrm{CH}_{2}\right.$ of thiadiazole), $1.19\left(\mathrm{t}, 3 \mathrm{H}, \mathrm{CH}_{3}\right.$ of thiadiazole), $9.59(\mathrm{~s}, 1 \mathrm{H}$, 
$\mathrm{OH}) ;{ }^{13} \mathrm{C}$ NMR (DMSO-d6): $\delta 171.4,161.2,159.9,155.8,140.3,136.9,134.8,116.6,115.2$, 22.9, 15.2; MS: $m / z\left(\mathrm{M}^{+}+1333.25\right)$.

\section{2-((5-ethyl-1,3,4-thiadiazol-2-yl)imino)-5-(4-methoxybenzylidene)thiazolidin-4-} one(4g): Yield: $71.43 \%$; m.p. 192-194 ${ }^{\circ} \mathrm{C}$; IR (KBr pellets) $\mathrm{cm}^{-1}: 1605.59$ (C=N str.), 1335.68 (C-N str.), 3553 (N-H str., thiazolidine ring), 1705 (C=O str., thiazolidin-4-one), 602.31 (C-S bend), 1603.21 (C=C str.), 2860.31 (C-H str., aliphatic), 3275.87 (C-H str., aromatic ring), 1227.31 \& 1092.43 (O- $\mathrm{CH}_{3}$ str., p-substitution on phenyl ring); ${ }^{1} \mathrm{H}$ NMR (400 MHz, DMSOd6): $\delta$ 6.92-7.76 (m, 4H, Ar-H), $12.04(\mathrm{~s}, 1 \mathrm{H}, \mathrm{NH}), 7.81(\mathrm{~s}, 1 \mathrm{H},-\mathrm{CH}=), 2.21\left(\mathrm{q}, 2 \mathrm{H}, \mathrm{CH}_{2}\right.$ of thiadiazole), $1.16\left(\mathrm{t}, 3 \mathrm{H}, \mathrm{CH}_{3}\right.$ of thiadiazole), $3.73\left(\mathrm{~s}, 3 \mathrm{H}, \mathrm{OCH}_{3}\right) ;{ }^{13} \mathrm{C} \mathrm{NMR}$ (DMSO-d 6$): \delta$ $169.8,155.6,154.4,141.8,140.6,129.8,124.4,114.8,112.6,43.8,23.2,14.9 ; \mathrm{MS}: \mathrm{m} / z\left(\mathrm{M}^{+}+1\right.$ 347.58).

2-((5-ethyl-1,3,4-thiadiazol-2-yl)imino)-5-(2-hydroxybenzylidene)thiazolidin-4one(4h): Yield: $73.24 \%$; m.p. $189-191^{\circ} \mathrm{C}$; IR (KBr pellets) $\mathrm{cm}^{-1}: 1636.36$ (C=N str.), 1306.40 (C-N str.), 3160 (N-H str., thiazolidine ring), 1721.13 (C=O str., thiazolidin-4-one), 644.72 (C$\mathrm{S}$ bend), 1611.28 (C=C str.), 3102.15 (C-H str., aliphatic), 3293.77 (C-H str., aromatic ring), 3550.12 (OH str.); ${ }^{1} \mathrm{H}$ NMR (400 MHz, DMSO-d6): $\delta$ 6.53-7.59 (m, 4H, Ar-H), 12.42 (s, 1H, $\mathrm{NH}), 7.93(\mathrm{~s}, 1 \mathrm{H},-\mathrm{CH}=), 2.18$ (q, $2 \mathrm{H}, \mathrm{CH}_{2}$ of thiadiazole), 1.24 (t, 3H, $\mathrm{CH}_{3}$ of thiadiazole), 10.19 (s, $1 \mathrm{H}, \mathrm{OH}) ;{ }^{13} \mathrm{C}$ NMR (DMSO-d $): \delta 171.2,155.5,153.3,152.3,139.4,129.9,126.4$, 125.8, 125.3, 118.8, 114.6, 22.8, 14.6; MS: $m / z\left(\mathrm{M}^{+}+1333.38\right)$.

\section{5-(4-(benzyloxy)benzylidene)-2-((5-ethyl-1,3,4-thiadiazol-2-yl)imino)thiazolidin-}

4-one(4i): Yield: $82.14 \%$; m.p. 244-246 ${ }^{\circ} \mathrm{C}$; IR (KBr pellets) $\mathrm{cm}^{-1}$ : 1602.74 (C=N str.), 1375.91 (C-N str.), 3448.19 (N-H str., thiazolidine ring), 1592.24 (C=O str., thiazolidin-4-one), 645.94 (C-S bend), 1507.89 (C=C str.), 2862.05 (C-H str., aliphatic), 3067.17 (C-H str., aromatic ring), $1232.32 \& 1099.82$ (C-O-C str., p-substitution on phenyl ring); ${ }^{1} \mathrm{H}$ NMR (400 MHz, DMSOd6): $\delta$ 6.72-7.79 (m, 9H, Ar-H), $12.23(\mathrm{~s}, 1 \mathrm{H}, \mathrm{NH}), 7.83(\mathrm{~s}, 1 \mathrm{H},-\mathrm{CH}=), 2.24$ (q, 2H, $\mathrm{CH}_{2}$ of thiadiazole), 1.27 (t, $3 \mathrm{H}, \mathrm{CH}_{3}$ of thiadiazole), $4.83\left(\mathrm{~s}, 2 \mathrm{H}, \mathrm{OCH}_{2}\right) ;{ }^{13} \mathrm{C}$ NMR (DMSO-d $): \delta$ 171.4, 155.8, 153.8, 150.6, 140.6, 130.6, 129.6, 126.2, 125.6, 125.4, 121.4, 115.4, 114.8, 68.4, 23.0, 14.9; $\mathrm{MS}: m / z\left(\mathrm{M}^{+}+1\right.$ 423.67).

\section{5-(3,4-dimethoxybenzylidene)-2-((5-ethyl-1,3,4-thiadiazol-2-yl)imino)thiazolidin-} 4-one (4j): Yield: $69.57 \%$; m.p. 201-203 ${ }^{\circ} \mathrm{C}$; IR (KBr pellets) $\mathrm{cm}^{-1}$ : 1611.18 (C=N str.), 1342.36 (C-N str.), 3552.02 (N-H str., thiazolidine ring), 1681.24 (C=O str., thiazolidin-4-one), 618.41 (C-S bend), 1596.64 (C=C str.), 3115.53 (C-H str., aliphatic), 3226.89 (C-H str., aromatic ring), $1286.77 \& 1109.21$ (C-O-C str., substitution on phenyl ring); ${ }^{1} \mathrm{H}$ NMR (400 MHz, DMSO-d6): $\delta$ 6.97-7.79 (m, 3H, Ar-H), 12.53 (s, 1H, NH), 7.78 (s, 1H, -CH=), 2.29 (q, $2 \mathrm{H}, \mathrm{CH}_{2}$ of thiadiazole), 1.21 (t, $3 \mathrm{H}, \mathrm{CH}_{3}$ of thiadiazole), 3.82 (s, $3 \mathrm{H}, \mathrm{OCH}_{3}$ of m-position), 3.80 (s, 3H, $\mathrm{OCH}_{3}$ of p-position); ${ }^{13} \mathrm{C}$ NMR (DMSO-d 6 ): $\delta 171.2,165.3,152.4,148.2,144.5$, 122.2, 120.4, 111.5, 111.2, 57.2, 22.1, 14.6; $\mathrm{MS}: m / z\left(\mathrm{M}^{+}+1\right.$ 377.38).

\section{2-((5-ethyl-1,3,4-thiadiazol-2-yl)imino)-5-(2-methoxybenzylidene)thiazolidin-4-}

one(4k): Yield: $72.37 \%$; m.p. $171-173^{\circ} \mathrm{C}$; IR (KBr pellets) $\mathrm{cm}^{-1}: 1605.03(\mathrm{C}=\mathrm{N}$ str.), 1337.05 (C-N str.), 3282.06 (N-H str., thiazolidine ring), 1710.82 ( $\mathrm{C}=\mathrm{O}$ str., thiazolidin-4-one), 610.07 (C-S bend), 1603.64 (C=C str.), 3014 (C-H str., aliphatic), 3105.45 (C-H str., aromatic ring), $1227.01 \& 1095.82$ (C-O-C str., o-substitution on phenyl ring); ${ }^{1} \mathrm{H}$ NMR (400 MHz, DMSOd6): $\delta$ 6.92-7.87 (m, 4H, Ar-H), $12.25(\mathrm{~s}, 1 \mathrm{H}, \mathrm{NH}), 7.93$ (s, 1H, -CH=), 2.19 (q, 2H, $\mathrm{CH}_{2}$ of thiadiazole), $1.30\left(\mathrm{t}, 3 \mathrm{H}, \mathrm{CH}_{3}\right.$ of thiadiazole), $3.88\left(\mathrm{~s}, 3 \mathrm{H}, \mathrm{OCH}_{3}\right) ;{ }^{13} \mathrm{C} \mathrm{NMR}$ (DMSO-d $): \delta$ 169.8, 165.4, 155.6, 155.4, 140.8, 121.8, 121.5, 116.6, 114.6, 111.6, 111.2, 52.9, 22.3, 14.2; MS: $m / z\left(\mathbf{M}^{+}+1347.23\right)$. 
2-((5-ethyl-1,3,4-thiadiazol-2-yl)imino)-5-(2-fluorobenzylidene)thiazolidin-4one(4l): Yield: $74.16 \%$; m.p. $261-263^{\circ} \mathrm{C}$; IR (KBr pellets) $\mathrm{cm}^{-1}: 1613.98$ (C=N str.), 1339.78 (C-N str.), 3492 (N-H str., thiazolidine ring), 1601.72 (C=O str., thiazolidin-4-one), 691.37 (C$\mathrm{S}$ bend), 1608.53 (C=C str.), 2850 (C-H str., aliphatic), 3117.32 (C-H str., aromatic ring), 1258.58 (C-F bend); ${ }^{1} \mathrm{H}$ NMR (400 MHz, DMSO-d 6$): \delta 7.02-7.58$ (m, 4H, Ar-H), $12.31(\mathrm{~s}, 1 \mathrm{H}$, $\mathrm{NH}), 7.95(\mathrm{~s}, 1 \mathrm{H},-\mathrm{CH}=), 2.24\left(\mathrm{q}, 2 \mathrm{H}, \mathrm{CH}_{2}\right.$ of thiadiazole $), 1.26\left(\mathrm{t}, 3 \mathrm{H}, \mathrm{CH}_{3}\right.$ of thiadiazole $) ;{ }^{13} \mathrm{C}$ NMR (DMSO-d $\mathrm{d}_{\text {): } \delta} \delta 171.6,165.4,155.8,149.4,140.6,127.9,126.4,122.4,120.8,116.6$, 115.2, 22.8, 14.6; MS: $m / z\left(\mathrm{M}^{+}+1335.19\right)$.

5-(3-bromobenzylidene)-2-((5-ethyl-1,3,4-thiadiazol-2-yl)imino)thiazolidin-4-one (4m): Yield: $84.47 \%$; m.p. $173-175^{\circ} \mathrm{C}$; IR (KBr pellets) $\mathrm{cm}^{-1}$ : 1616.48 (C=N str.), 1337.54 (C$\mathrm{N}$ str.), 3277.27 (N-H str., thiazolidine ring), 1636.36 (C=O str., thiazolidin-4-one), 691.01 (C$\mathrm{S}$ bend), 1600.80 (C=C str.), 2862 (C-H str., aliphatic), 3117.04 (C-H str., aromatic ring), 532.45 (C-Br bend); ${ }^{1} \mathrm{H}$ NMR (400 MHz, DMSO-d $): \delta 7.12-7.86(\mathrm{~m}, 4 \mathrm{H}, \mathrm{Ar}-\mathrm{H}), 12.24(\mathrm{~s}, 1 \mathrm{H}$, $\mathrm{NH}), 7.81$ (s, $1 \mathrm{H},-\mathrm{CH}=), 2.23$ (q, $2 \mathrm{H}, \mathrm{CH}_{2}$ of thiadiazole), 1.25 (t, $3 \mathrm{H}, \mathrm{CH}_{3}$ of thiadiazole); ${ }^{13} \mathrm{C}$ NMR (DMSO-d6): $\delta$ 170.6, 165.4, 153.9, 139.9, 135.2, 126.5, 125.4, 123.6, 121.3, 115.4, 21.9, 13.9; MS: $m / z\left(\mathrm{M}^{+}+1396.01\right)$.

\section{5-((4-dimethylamino)benzylidene)-2-((5-ethyl-1,3,4-thiadiazol-2-}

yl)imino)thiazolidin-4-one(4n): Yield: $78.14 \%$; m.p. 264-266 ${ }^{\circ} \mathrm{C}$; IR (KBr pellets) $\mathrm{cm}^{-1}$ : 1602.39 ( $\mathrm{C}=\mathrm{N}$ str.), 1306.26 (C-N str.), 3292.52 ( $\mathrm{N}-\mathrm{H}$ str., thiazolidine ring), 1734.26 (C=O str., thiazolidin-4-one), 645.01 (C-S bend), 1532.86 (C=C str.), 2946.77 (C-H str., aliphatic), 3114.84 (C-H str., aromatic ring); ${ }^{1} \mathrm{H}$ NMR (400 MHz, DMSO-d 6 ): $\delta$ 6.77-7.79 (m, 4H, Ar-H), 12.49 (s, 1H, NH), 7.80 (s, 1H, -CH=), 2.43 (q, 2H, $\mathrm{CH}_{2}$ of thiadiazole), 1.27 (t, 3H, $\mathrm{CH}_{3}$ of thiadiazole), 2.98 (s, 6H, $2 \times \mathrm{CH}_{3}$ ); ${ }^{13} \mathrm{C}$ NMR (DMSO-d6): $\delta$ 172.1, 166.2, 153.5, 151.4, 140.4, 131.2, 125.2, 112.5, 111.2, 42.4, 23.1, 14.4; MS: $m / z\left(\mathrm{M}^{+}+1360.39\right)$.

\section{2-((5-ethyl-1,3,4-thiadiazol-2-yl)imino)-5-(4-methylbenzylidene)thiazolidin-4-}

one(4o): Yield: $73.56 \%$; m.p. $195-197^{\circ} \mathrm{C}$; IR (KBr pellets) $\mathrm{cm}^{-1}: 1629.75$ (C=N str.), 1336.70 (C-N str.), 3411.76 (N-H str., thiazolidine ring), 1713.33 (C=O str., thiazolidin-4-one), 681.27 (C-S bend), 1601.44 (C=C str.), 2986.35 (C-H str., aliphatic), 3053.41 (C-H str., aromatic ring); ${ }^{1} \mathrm{H}$ NMR (400 MHz, DMSO-d6): $\delta$ 7.19-7.82 (m, 4H, Ar-H), 12.35 (s, 1H, NH), 7.84 (s, 1H, $\mathrm{CH}=), 2.36\left(\mathrm{q}, 2 \mathrm{H}, \mathrm{CH}_{2}\right.$ of thiadiazole), 1.29 (t, 3H, $\mathrm{CH}_{3}$ of thiadiazole), $2.37\left(\mathrm{~s}, 3 \mathrm{H}, \mathrm{CH}_{3}\right.$ adjacent to phenyl ring); ${ }^{13} \mathrm{C}$ NMR (DMSO-d6): $\delta 169.8,166.6,154.8,141.5,139.6,138.4$, 136.2, 135.8, 114.1, 23.4, 20.9, 14.2; MS: $m / z\left(\mathrm{M}^{+}+1331.36\right)$.

2-((5-ethyl-1,3,4-thiadiazol-2-yl)imino)-5-(3-hydroxybenzylidene)thiazolidin-4one(4p): Yield: $71.18 \%$; m.p. 202-204 ${ }^{\circ} \mathrm{C}$; IR (KBr pellets) $\mathrm{cm}^{-1}: 1608.39$ (C=N str.), 1341.33 (C-N str.), 3160.18 (N-H str., thiazolidine ring), 1776.22 (C=O str., thiazolidin-4-one), 617.99 (C-S bend), 1596.16 (C=C str.), 2995 (C-H str., aliphatic), 3105.15 (C-H str., aromatic ring), 3422.29 (OH str.); ${ }^{1} \mathrm{H}$ NMR (400 MHz, DMSO-d6): $\delta$ 6.57-7.59 (m, 4H, Ar-H), 12.19 (s, 1H, $\mathrm{NH}), 7.79$ (s, 1H, - $\mathrm{CH}=), 2.32$ (q, $2 \mathrm{H}, \mathrm{CH}_{2}$ of thiadiazole), 1.23 (t, 3H, $\mathrm{CH}_{3}$ of thiadiazole), 9.49 (s, 1H, OH); ${ }^{13} \mathrm{C}$ NMR (DMSO-d6): $\delta 171.8,164.6,154.8,154.6,141.5,140.8,134.6$, 126.7, 118.2, 116.6, 115.6, 22.8, 14.6; MS: $m / z\left(\mathrm{M}^{+}+1333.24\right)$.

\section{2-((5-ethyl-1,3,4-thiadiazol-2-yl)imino)-5-(2-nitrobenzylidene)-thiazolidin-4-}

one(4q): Yield: $68.89 \%$; m.p. $182-184^{\circ} \mathrm{C}$; IR (KBr pellets) $\mathrm{cm}^{-1}: 1602.79$ (C=N str.), 1335.72 (C-N str.), 3275.68 (N-H str., thiazolidine ring), 1704.23 (C=O str., thiazolidin-4-one), 602.05 (C-S bend), 1595.71 (C=C str.), 3014 (C-H str., aliphatic), 3120.25 (C-H str., aromatic ring), 1490.83 ( $\mathrm{NO}_{2}$ assym. str.), 1291.45 ( $\mathrm{NO}_{2}$ symm. Str.) ; ${ }^{1} \mathrm{H}$ NMR (400 MHz, DMSO-d $)$ ): $\delta$ 7.57-7.93 (m, 4H, Ar-H), $12.27(\mathrm{~s}, 1 \mathrm{H}, \mathrm{NH}), 8.28(\mathrm{~s}, 1 \mathrm{H},-\mathrm{CH}=), 2.26\left(\mathrm{q}, 2 \mathrm{H}, \mathrm{CH}_{2}\right.$ of 
thiadiazole), $1.30\left(\mathrm{t}, 3 \mathrm{H}, \mathrm{CH}_{3}\right.$ of thiadiazole); ${ }^{13} \mathrm{C}$ NMR (DMSO-d6): $\delta 171.8,162.4,154.6$, 153.5, 134.8, 126.5, 117.8, 116.7, 115.4, 21.8, 12.9; MS: $\mathrm{m} / z\left(\mathrm{M}^{+}+1362.28\right)$.

\section{5-(3-chlorobenzylidene)-2-((5-ethyl-1,3,4-thiadiazol-2-yl)imino)thiazolidin-4-}

one(4r): Yield: $75.73 \%$; m.p. 219-221 ${ }^{\circ} \mathrm{C}$; IR ( $\mathrm{KBr}$ pellets) $\mathrm{cm}^{-1}: 1636.36$ (C=N str.), 1166.47 (C-N str.), 3494.18 (N-H str., thiazolidine ring), 1726.15 (C=O str., thiazolidin-4-one), 691.01 (C-S bend), 1601 (C=C str.), 2940.23 (C-H str., aliphatic), 3117.04 (C-H str., aromatic ring), 764.57 (C-Cl bend) ; ${ }^{1} \mathrm{H}$ NMR (400 MHz, DMSO-d6): $\delta$ 7.09-7.68 (m, 4H, Ar-H), 12.45 (s, $1 \mathrm{H}, \mathrm{NH}), 7.82$ (s, 1H, $-\mathrm{CH}=), 2.32$ (q, $2 \mathrm{H}, \mathrm{CH}_{2}$ of thiadiazole), 1.28 (t, 3H, $\mathrm{CH}_{3}$ of thiadiazole); ${ }^{13}$ C NMR (DMSO-d6): $\delta 170.8,162.9,156.4,140.7,127.4,125.6,121.6,119.6,117.8,117.5$, 115.4, 22.5, 13.4; MS: $m / z\left(\mathrm{M}^{+}+1351.92\right)$.

\section{5-(2-chlorobenzylidene)-2-((5-ethyl-1,3,4-thiadiazol-2-yl)imino)thiazolidin-4-}

one(4s): Yield: 72.39\%; m.p. 227-229 ${ }^{\circ}$; IR (KBr pellets) $\mathrm{cm}^{-1}$ : 1702 (C=N str.), 1342.36 (C$\mathrm{N}$ str.), 3492 (N-H str., thiazolidine ring), 1611.98 (C=O str., thiazolidin-4-one), 702.17 (C-S bend), 1596.64 (C=C str.), 3115.53 (C-H str., aliphatic), 3226.89 (C-H str., aromatic ring), 755.77 (C-Cl bend) ; ${ }^{1} \mathrm{H}$ NMR (400 MHz, DMSO-d6): $\delta$ 6.91-7.86 (m, 4H, Ar-H), 12.32 (s, $1 \mathrm{H}, \mathrm{NH}), 7.93$ (s, $1 \mathrm{H},-\mathrm{CH}=), 2.28$ (q, $2 \mathrm{H}, \mathrm{CH}_{2}$ of thiadiazole), 1.25 (t, 3H, $\mathrm{CH}_{3}$ of thiadiazole); ${ }^{13} \mathrm{C}$ NMR (DMSO-d6): $\delta$ 166.5, 165.6, 154.4, 147.8, 145.6, 141.4, 137.4, 135.8, 134.7, 120.6, 22.9, 12.7; MS: $m / z\left(\mathrm{M}^{+}+1351.93\right)$.

\section{5-(2-bromobenzylidene)-2-((5-ethyl-1,3,4-thiadiazol-2-yl)imino)thiazolidin-4-}

one(4t):Yield: $75.58 \%$; m.p. $192-194^{\circ} \mathrm{C}$; IR (KBr pellets) $\mathrm{cm}^{-1}: 1611.18$ (C=N str.), 1232.46 (C-N str.), 3495 (N-H str., thiazolidine ring), 1702.23 (C=O str., thiazolidin-4-one), 645.70 (C$\mathrm{S}$ bend), 1602.74 (C=C str.), 3108.43 (C-H str., aliphatic), 3020 (C-H str., aromatic ring), 535.19 (C-Br bend) ; ${ }^{1} \mathrm{H}$ NMR (400 MHz, DMSO-d6): $\delta$ 6.74-7.79 (m, 4H, Ar-H), 12.28 (s, $1 \mathrm{H}, \mathrm{NH}), 7.89$ (s, 1H, - $\mathrm{CH}=), 2.22$ (q, $2 \mathrm{H}, \mathrm{CH}_{2}$ of thiadiazole), 1.21 (t, 3H, $\mathrm{CH}_{3}$ of thiadiazole); ${ }^{13} \mathrm{C}$ NMR (DMSO-d6): $\delta 171.8,161.4,155.4,150.5,142.6,140.8,135.9,135.6,131.4,119.6$, 23.2, 13.6; MS: $m / z\left(\mathrm{M}^{+}+1\right.$ 396.08).

\subsection{The spectrophotometric method to determine the AChE enzyme activity.}

Ellman's method was used to determine AChE activity by using a spectrophotometer $[43,44]$. The inhibitory activities ( $\mathrm{IC}_{50}$ values) of synthesized compounds (4a-t) were calculated with donepezil as a reference compound. The test tube was filled with $2.5 \mathrm{ml}$ of 0.05 $\mathrm{M}$ phosphate buffer $\mathrm{pH} 7.2,357 \mu \mathrm{l}$ of test solution in dimethyl sulfoxide (DMSO) at various concentrations $(12.5-200 \mu \mathrm{M} / \mathrm{ml}), 357 \mu \mathrm{l} 2.5 \mathrm{U} / \mathrm{ml} \mathrm{AChE}$, and $357 \mu 1$ of $0.01 \mathrm{M}$ of DTNB $(5,5-$ dithio-bis-(2-nitrobenzoic acid) in the buffer. Donepezil was used as a positive control and was prepared the same way as the test by dissolving in $0.05 \mathrm{M}$ phosphate buffer $\mathrm{pH} 7.2$ in serial concentrations. For fifteen minutes, the mixture was incubated at $37^{\circ} \mathrm{C}$. Substrate (acetylthiocholine iodide $0.075 \mathrm{M}$ ) in buffer was added after incubation. The absorbance of the yellow anion was measured at $412 \mathrm{~nm}$ for 5 minutes. Instead of using enzyme solution, a blank was prepared using the buffer. $\left(\mathrm{A}_{0}-\mathrm{A}_{1} / \mathrm{A}_{0}\right) \mathrm{x} 100$ was used to measure the percent inhibition of enzyme activity, where $A_{0}$ represented the absorbance of the control and $A_{1}$ represented the absorbance of the sample. This test was performed in triplicate. By performing a linear regression analysis between the inhibition percentage and the concentration of test compounds that inhibits substrate hydrolysis by $50 \%$ (IC50) was calculated using Excel software. In terms of $\mathrm{pIC}_{50}(\mathrm{mM})$, the results were expressed as a mean $\pm \mathrm{SD}$ of three different experiments. 


\section{Results and Discussion}

\subsection{Chemistry.}

Title compounds 2,5-disubstituted-4-thiazolidinones 4(a-t) were synthesized from the reactions of Schiff bases( III) with different benzaldehyde and anhydrous sodium acetate. The appropriate Schiff bases III were prepared by reacting thiadiazole thiourea (II) with chloroacetic acid, while compound II was synthesized by reacting ammonium thiocyanate and dilute $\mathrm{HCl}$. The reaction of propanoic acid and thiosemicarbazide in the presence of concentrated sulphuric acid yielded the required compound I, as shown in Scheme 1. The physicochemical properties of the synthesized derivatives 4(a-t) are listed in Table 1, were obtained in appreciable yield. The synthesized derivatives $4(a-t)$ were confirmed by their spectral data.

Table 1. Characterization of synthesized compounds (4(a-t)) in terms of their physicochemical properties.

\begin{tabular}{l|l|l|l|l|l} 
Compound & Mol. Formula & Mol.Weight & Melting Point $\left({ }^{\circ} \mathbf{C}\right)$ & $\mathbf{R}_{\mathbf{f}}$ Value & \% Yield \\
\hline $\mathbf{4 a}$ & $\mathrm{C}_{14} \mathrm{H}_{12} \mathrm{~N}_{4} \mathrm{OS}_{2}$ & 316.40 & $208-210$ & 0.73 & 83.67 \\
\hline $\mathbf{4 b}$ & $\mathrm{C}_{14} \mathrm{H}_{11} \mathrm{ClN}_{4} \mathrm{OS}_{2}$ & 350.84 & $240-242$ & 0.79 & 79.65 \\
\hline $\mathbf{4 c}$ & $\mathrm{C}_{14} \mathrm{H}_{11} \mathrm{BrN}_{4} \mathrm{OS}_{2}$ & 395.29 & $241-243$ & 0.81 & 84.28 \\
\hline $\mathbf{4 d}$ & $\mathrm{C}_{14} \mathrm{H}_{11} \mathrm{~N}_{5} \mathrm{O}_{3} \mathrm{~S}_{2}$ & 361.39 & $203-205$ & 0.78 & 72.57 \\
\hline $\mathbf{4 e}$ & $\mathrm{C}_{14} \mathrm{H}_{11} \mathrm{FN}_{4} \mathrm{OS}_{2}$ & 334.39 & $250-252$ & 0.74 & 81.23 \\
\hline $\mathbf{4 f}$ & $\mathrm{C}_{14} \mathrm{H}_{12} \mathrm{~N}_{4} \mathrm{O}_{2} \mathrm{~S}_{2}$ & 332.40 & $211-213$ & 0.71 & 76.57 \\
\hline $\mathbf{4 g}$ & $\mathrm{C}_{15} \mathrm{H}_{14} \mathrm{~N}_{4} \mathrm{O}_{2} \mathrm{~S}_{2}$ & 346.42 & $192-194$ & 0.72 & 71.43 \\
\hline $\mathbf{4 h}$ & $\mathrm{C}_{14} \mathrm{H}_{12} \mathrm{~N}_{4} \mathrm{O}_{2} \mathrm{~S}_{2}$ & 332.40 & $189-191$ & 0.69 & 73.24 \\
\hline $\mathbf{4 i}$ & $\mathrm{C}_{21} \mathrm{H}_{18} \mathrm{~N}_{4} \mathrm{O}_{2} \mathrm{~S}_{2}$ & 422.52 & $244-246$ & 0.83 & 82.14 \\
\hline $\mathbf{4 j}$ & $\mathrm{C}_{16} \mathrm{H}_{16} \mathrm{~N}_{4} \mathrm{O}_{3} \mathrm{~S}_{2}$ & 376.45 & $201-203$ & 0.70 & 69.57 \\
\hline $\mathbf{4 k}$ & $\mathrm{C}_{15} \mathrm{H}_{14} \mathrm{~N}_{4} \mathrm{O}_{2} \mathrm{~S}_{2}$ & 346.42 & $171-173$ & 0.71 & 72.37 \\
\hline $\mathbf{4 l}$ & $\mathrm{C}_{14} \mathrm{H}_{11} \mathrm{FN}_{4} \mathrm{OS}_{2}$ & 334.39 & $261-263$ & 0.73 & 74.16 \\
\hline $\mathbf{4 m}$ & $\mathrm{C}_{14} \mathrm{H}_{11} \mathrm{BrN}_{4} \mathrm{OS}_{2}$ & 395.29 & $173-175$ & 0.82 & 84.47 \\
\hline $\mathbf{4 n}$ & $\mathrm{C}_{16} \mathrm{H}_{17} \mathrm{~N}_{5} \mathrm{OS}_{2}$ & 359.47 & $264-266$ & 0.75 & 78.14 \\
\hline $\mathbf{4 o}$ & $\mathrm{C}_{15} \mathrm{H}_{14} \mathrm{~N}_{4} \mathrm{OS}_{2}$ & 330.42 & $195-197$ & 0.78 & 73.56 \\
\hline $\mathbf{4 p}$ & $\mathrm{C}_{14} \mathrm{H}_{12} \mathrm{~N}_{4} \mathrm{O}_{2} \mathrm{~S}_{2}$ & 332.40 & $202-204$ & 0.72 & 71.18 \\
\hline $\mathbf{4 q}$ & $\mathrm{C}_{14} \mathrm{H}_{11} \mathrm{~N}_{5} \mathrm{O}_{3} \mathrm{~S}_{2}$ & 361.39 & $182-184$ & 0.81 & 68.89 \\
\hline $\mathbf{4 r}$ & $\mathrm{C}_{14} \mathrm{H}_{11} \mathrm{ClN}_{4} \mathrm{OS}_{2}$ & 350.84 & $219-221$ & 0.80 & 75.73 \\
\hline $\mathbf{4 s}$ & $\mathrm{C}_{14} \mathrm{H}_{11} \mathrm{ClN}_{4} \mathrm{OS}_{2}$ & 350.84 & $227-229$ & 0.76 & 72.39 \\
\hline $\mathbf{4 t}$ & $\mathrm{C}_{14} \mathrm{H}_{11} \mathrm{BrN}_{4} \mathrm{OS}_{2}$ & 395.29 & $192-194$ & 0.83 & 75.58
\end{tabular}

The absorption bands for $\mathrm{C}=\mathrm{N}, \mathrm{C}-\mathrm{N}, \mathrm{C}-\mathrm{S}$, and $\mathrm{C}=\mathrm{C}$ stretching at 1702-1602, 1384$1166,702-602$, and $1627-1507 \mathrm{~cm}^{-1}$ were found in the IR spectra of final derivatives $4(\mathrm{a}-\mathrm{t})$, respectively. The absorption bands in the range 3562-3116 and $1799-1601 \mathrm{~cm}^{-1}$ in the IR spectra of the compounds were assigned to the $\mathrm{N}-\mathrm{H}$ and $\mathrm{C}=\mathrm{O}$ stretching of the thiazolidinone nucleus, respectively. At 3293-2924 and 3115-2850 $\mathrm{cm}^{-1}$, aromatic and aliphatic C-H stretching vibrations were observed, respectively. At $1490-1438 \mathrm{~cm}^{-1}$, asymmetric nitro group stretching vibrations were observed, while symmetric nitro group stretching vibrations were observed at 1384-1291 $\mathrm{cm}^{-1}$. At 1286-1227 and 1109-1092 $\mathrm{cm}^{-1}$, the C-O-C stretching vibrations appeared. $\mathrm{C}-\mathrm{Cl}$ stretching vibrations have a strong band at $764-752 \mathrm{~cm}^{-1}$ that has been assigned to them. IR vibrations at 535-532 and $1258-1227 \mathrm{~cm}^{-1}$ indicate the presence of the bromo and fluoro groups, respectively. IR vibrations at $3550-3422 \mathrm{~cm}^{-1}$ suggested the presence of an $\mathrm{OH}$ group in the synthesized derivatives.

Singlets for the $-\mathrm{CH}=$ and $-\mathrm{NH}$ protons were observed in the ${ }^{1} \mathrm{H}$ NMR spectra of compounds (400 MHz in DMSO) at 7.72-8.28 and 12.04-12.53 ppm, respectively. For thiadiazole adjacent $-\mathrm{CH}_{2}$ and $-\mathrm{CH}_{3}$, quartet, and triplet signals appeared around 2.13-2.43 and 1.16-1.29 ppm, respectively. Between 6.52 and $8.38 \mathrm{ppm}$, aromatic protons appeared as a 
multiplet. Other protons' signals appeared at the predicted chemical shifts. The ${ }^{13} \mathrm{C}$ NMR signals appeared at the chemical shifts that were predicted. $\mathrm{M}^{+}+1$ peaks were found in the mass of the synthesized compounds.

\subsection{AChE inhibition evaluation.}

The inhibitory activities of synthesized compounds 4a-t, against AChE (from Electrophorus electricus) were determined using a spectrophotometer using Ellman's method and donepezil as a reference compound. Table 2 shows the $\mathrm{AChE}$ inhibition pIC50 values of the synthesized derivatives.

These findings revealed that all compounds have AChE inhibitory activity and successfully inhibited the enzyme. The most effective inhibitors were compounds $4 \mathrm{~d}, 4 \mathrm{~g}, 4 \mathrm{i}$, $4 \mathrm{j}, 4 \mathrm{n}$, and $4 \mathrm{o}$, with $\mathrm{pIC}_{50}$ values of $1.01 \pm 0.010,1.04 \pm 0.013,1.22 \pm 0.002,1.19 \pm 0.021$, $1.04 \pm 0.017$, and $1.30 \pm 0.007 \mathrm{mM}$.

Compared to donepezil and other synthesized compounds, compound 4o with a methyl substituent at the para position showed potent inhibition of AChE with a pIC50 value of $1.30 \pm 0.007 \mathrm{mM}$. Compound $4 \mathrm{i}(\mathrm{pIC} 50=1.22 \pm 0.002 \mathrm{mM}$ ), which has a benzyloxy substituent at the para position, was the second most potent. The replacement of the chloro group with the fluoro group in compounds $4 \mathrm{~b}$ and $4 \mathrm{e}$ decreased $\mathrm{AChE}$ inhibitory activity, with pIC 50 values of $0.88 \pm 0.003$ and $0.84 \pm 0.013 \mathrm{mM}$, respectively.

Table 2. In vitro $\mathrm{AChE}$ inhibition $\left(\mathrm{pIC}_{50}, \mathrm{mM}\right)$ of the synthesized compounds (4(a-t)).

\begin{tabular}{l|l} 
Compound & AChE $\mathbf{~ I C}_{\mathbf{5 0}}(\mathbf{m M})$ \\
\hline $\mathbf{4 a}$ & $0.98 \pm 0.012$ \\
\hline $\mathbf{4 b}$ & $0.88 \pm 0.003$ \\
\hline $\mathbf{4 c}$ & $0.91 \pm 0.064$ \\
\hline $\mathbf{4 d}$ & $1.01 \pm 0.010$ \\
\hline $\mathbf{4 e}$ & $0.84 \pm 0.013$ \\
\hline $\mathbf{4 f}$ & $0.90 \pm 0.017$ \\
\hline $\mathbf{4 g}$ & $1.04 \pm 0.013$ \\
\hline $\mathbf{4 h}$ & $0.71 \pm 0.005$ \\
\hline $\mathbf{4 i}$ & $1.22 \pm 0.002$ \\
\hline $\mathbf{4 j}$ & $1.19 \pm 0.021$ \\
\hline $\mathbf{4 k}$ & $0.83 \pm 0.023$ \\
\hline $\mathbf{4 l}$ & $0.78 \pm 0.009$ \\
\hline $\mathbf{4 m}$ & $0.75 \pm 0.005$ \\
\hline $\mathbf{4 n}$ & $1.04 \pm 0.017$ \\
\hline $\mathbf{4 o}$ & $1.30 \pm 0.007$ \\
\hline $\mathbf{4 p}$ & $0.78 \pm 0.028$ \\
\hline $\mathbf{4 q}$ & $0.76 \pm 0.015$ \\
\hline $\mathbf{4 r}$ & $0.83 \pm 0.012$ \\
\hline $\mathbf{4 s}$ & $0.76 \pm 0.018$ \\
\hline $\mathbf{4 t}$ & $0.75 \pm 0.015$ \\
\hline $\mathbf{D o n e p e z i l}$ & $1.41 \pm 0.014$
\end{tabular}

The activity is reduced by replacing the para chloro in compound $4 \mathrm{~b}$ with a chloro $(\mathrm{Cl})$ group at the ortho position (in compound 4s). The chloro substituent exerted greater AChE inhibitory activity than the fluoro substituent, according to the results. The compound $4 \mathrm{o}$ with the methyl group had higher AChE inhibitory activity with a pIC50 value of $1.30 \pm 0.007 \mathrm{mM}$ than the methoxy-substituted compound $(4 \mathrm{~g})$ with a $\mathrm{pIC}_{50}$ value of $1.04 \pm 0.013 \mathrm{mM}$. The compound $4 \mathrm{~d}$ with the nitro group at the para position showed a higher $\mathrm{pIC}_{50}$ value of $1.01 \pm 0.010 \mathrm{mM}$ than the nitro group at the ortho position, having a pIC50 value of $0.76 \pm 0.015$ $\mathrm{mM}$. The AChE inhibitory activity is reduced when the para bromo group (4c) with a pIC50 value of $0.91 \pm 0.064 \mathrm{mM}$ is replaced with ortho and meta $(4 \mathrm{t}, 4 \mathrm{~m})$ with pIC50 values of 
$0.75 \pm 0.015$ and $0.75 \pm 0.005 \mathrm{mM}$, respectively. The results indicated that these new analogs' AChE inhibitory activity had been greatly influenced. A closer examination of the test compounds' structures revealed that the substituent modulates their AChE inhibitory function. As a result, these synthesized derivatives are being used as a pharmacophore to develop new and more effective acetylcholinesterase inhibitors.

\subsection{Structure-activity relationship.}

The following structure-activity relationship can be taken from acetylcholinesterase inhibitor studies (Figure 1):

- The different benzaldehyde substitutions used to synthesize the final derivatives (4a-t) significantly impacted the acetylcholinesterase inhibitor function. The acetylcholinesterase inhibitor activity was increased by substituting electron releasing groups; methyl $\left(-\mathrm{CH}_{3}\right)$ and benzyloxy at the para position in the synthesized compounds 40 and $4 \mathrm{i}$. These compounds may also be used as drug candidates to developing new acetylcholinesterase inhibitors that are more active and less toxic.

- When a bromo group (-Br) was substituted at the para position of the synthesized compound (4c), with a chloro group (-Cl) was substituted at the para position (4b), the acetylcholinesterase inhibitor activity was increased.

- In the synthesized compounds, replacing the chloro group (-Cl) with the fluoro group (-F) reduced the acetylcholinesterase inhibitor activity.

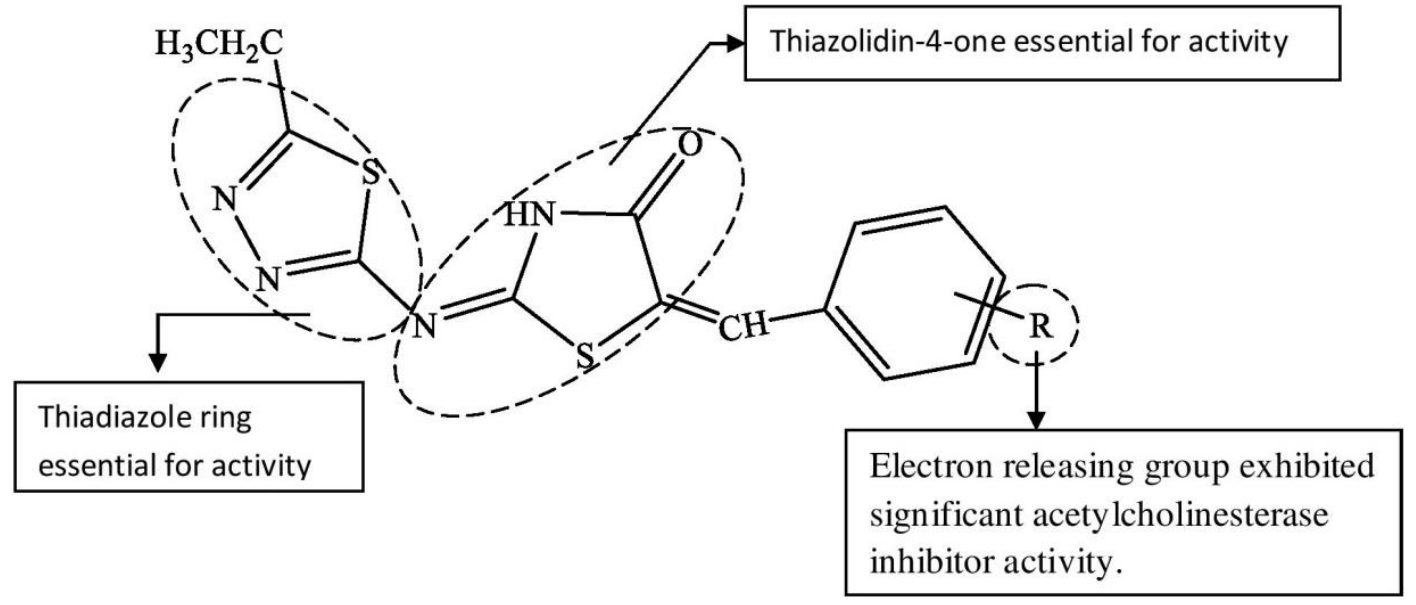

Figure 1. The structure and activity Relationship of the synthesized derivatives.

\section{Conclusions}

A set of thiazolidine-4-one derivatives (4a-4t) were synthesized and analyzed by IR, ${ }^{1} \mathrm{H}$ NMR, ${ }^{13} \mathrm{C}$ NMR, and mass spectral techniques and then evaluated for their ability to inhibit AChE. The inhibitory activities of synthesized derivatives $4 a-4 t$ against AChE (from Electrophorus electricus (C3389)) were determined using a spectrophotometer using Ellman's method and donepezil as a reference compound. The synthetic derivatives' AChE inhibition data ( $\mathrm{pIC}_{50}$ value) were calculated. The biological screening showed that all of the compounds have AChE inhibitory activity and have inhibited the enzyme successfully. The AChE enzyme inhibitors $4 \mathrm{o}$ and $4 \mathrm{i}$, which have an electron-donating group substitution on the aromatic ring, were found to be potent. This would be a better candidate for further research, and it could be tested to enhance further discoveries of AChE inhibitors. 


\section{Funding}

This research received no external funding.

\section{Acknowledgments}

The authors thank and greatly acknowledge the Chairman, Department of Pharmaceutical Sciences, Guru Jambheshwar University of Science and Technology, Hisar, for providing necessary facilities and consistent support. Our sincere thanks to Dr. A.P.J. Abdul Kalam, Central Instrumentation Laboratory, GJUS\&T, Hisar, Haryana, India, for spectral analysis.

\section{Conflicts of Interest}

The authors declare no conflict of interest.

\section{References}

1. Weller, J.; Budson, A. Current understanding of alzheimer's disease diagnosis and treatment. F1000Research 2018, 7, 1161, https://doi.org/10.12688/f1000research.14506.1.

2. Skrzypek, A.; Matysiak, J.; Niewiadomy, A.; Bajda, M.; Szymanski, P. Synthesis and biological evaluation of 1,3,4-thiadiazole analogues as novel AChE and BuChE inhibitors. Eur. J. Med. Chem. 2013, 62, 311-319, https://doi.org/10.1016/j.ejmech.2012.12.060.

3. Fish, P.V.; Steadman, D.; Bayle, E. D.; Whiting, P. New approaches for the treatment of alzheimer'sdisease. Bioorg. Med. Chem. Lett. 2019, 29, 125-133, https://doi.org/10.1016/j.bmcl.2018.11.034.

4. Dhanasekaran, S.; Perumal, P.; Palayan, M. In-vitro screening for acetylcholinesterase enzyme inhibition potential and antioxidant activity of extracts of ipomoea aquatica forsk: therapeutic lead for alzheimer's disease. J. Appl. Pharm. Sci.2015, 012-016, https://doi.org/10.7324/JAPS.2015.50203.

5. Berwaldt, G. A.; Gouvea, D. P.; da Silva, D. S.; das Neves, A. M.; Soares, M. S. P.; Azambuja, J. H.; Siqueira, G. M.; Spanevello, R. M.; Cunico, W. Synthesis and biological evaluation of benzothiazin-4-ones: a possible new class of acetylcholinesterase inhibitors. J. Enzyme Inhib. Med. Chem. 2019, 34, 196-202, https://doi.org/10.1080/14756366.2018.1543286.

6. Ujan, R.; Saeed, A.; Channar, P.; Larik, F.; Abbas, Q.; Alajmi, M.; El-Seedi, H.; Rind, M.; Hassan, M.; Raza, H.; Seo, S.-Y. Drug-1,3,4-thiadiazole conjugates as novel mixed-type inhibitors of acetylcholinesterase: synthesis, molecular docking, pharmacokinetics, and admet evaluation. Molecules 2019, 24, 860, https://doi.org/10.3390/molecules24050860.

7. Aisha; Raza, M. A.; Sumrra, S. H.; Javed, K.; Saqib, Z.; Maurin, J. K.; Budzianowski, A. Synthesis, characterization and molecular modeling of amino derived thiazolidinones as esterase and glucosidase inhibitors. J. Mol. Struct. 2020, 1219, 128609, https://doi.org/10.1016/j.molstruc.2020.128609.

8. Shehzadi, S. A.; Khan, I.; Saeed, A.; Larik, F. A.; Channar, P. A.; Hassan, M.; Raza, H.; Abbas, Q.; Seo, S.Y. One-pot four-component synthesis of thiazolidin-2-imines using cui/znii dual catalysis: a new class of acetylcholinesterase inhibitors. Bioorganic Chem. 2019, 84, 518-528, https://doi.org/10.1016/j.bioorg.2018.12.002.

9. Maccari, R.; Corso, A. D.; Giglio, M.; Moschini, R.; Mura, U.; Ottana, R. In vitro evaluation of 5-arylidene2-thioxo-4-thiazolidinones active as aldose reductase inhibitors. Bioorg. Med. Chem. Lett. 2011, 21, 200203, https://doi.org/10.1016/j.bmcl.2010.11.041.

10. Rahim, F.; Taha, M.; Ullah, H.; Wadood, A.; Selvaraj, M.; Rab, A.; Sajid, M.; Shah, S. A. A.; Uddin, N.; Gollapalli, M. Synthesis of new arylhydrazide bearing schiff bases/thiazolidinone: $\alpha$-amylase, urease activities and their molecular docking studies. Bioorganic Chem. 2019, 91, 103112, https://doi.org/10.1016/j.bioorg.2019.103112.

11. Holota, S.; Kryshchyshyn, A.; Derkach, H.; Trufin, Y.; Demchuk, I.; Gzella, A.; Grellier, P.; Lesyk, R. Synthesis of 5-enamine-4-thiazolidinone derivatives with trypanocidal and anticancer activity. Bioorganic Chem. 2019, 86, 126-136, https://doi.org/10.1016/j.bioorg.2019.01.045.

12. Tahmasvand, R.; Bayat, P.; Vahdaniparast, S. M.; Dehghani, S.; Kooshafar, Z.; Khaleghi, S.; Almasirad, A.; Salimi, M. Design and synthesis of novel 4-thiazolidinone derivatives with promising anti-breast cancer 
activity: synthesis, characterization, in vitro and in vivo results. Bioorganic Chem. 2020, 104, 104276, https://doi.org/10.1016/j.bioorg.2020.104276.

13. Kobylinska, L.; Lozynskii, A.; Lesyk, R.; Stoika, R.; Vari, S. G. Biodistribution and anticancer characteristics of les-3833, a novel 4-thiazolidinone-based lead compound. Sci. Pharm. 2020, 88, 18, https://doi.org/10.3390/scipharm88020018.

14. Gupta, A.; Singh, R.; Sonar, P. K.; Saraf, S. K. Novel 4-thiazolidinone derivatives as anti-infective agents: synthesis, characterization, and antimicrobial evaluation. Biochem. Res. Int. 2016, 2016, 1-8, https://doi.org/10.1155/2016/8086762.

15. Dincel, E. D.; Ulusoy- Guzeldemirci, N.; Şatana, D.; Kucukbasmacı, O. Design, synthesis, characterization and antimicrobial evaluation of some novel hydrazinecarbothioamide, 4-thiazolidinone and 1,2,4-triazole-3thione derivatives. J. Heterocycl. Chem. 2021, 58, 195-205, https://doi.org/10.1002/jhet.4159.

16. Kulkarni, P. S.; Karale, S. N.; Khandebharad, A. U.; Agrawal, B. R.; Sarda, S. R. Design, synthesis, and biological evaluation of newer arylidene incorporated 4-thiazolidinones derivatives as potential antimicrobial agents. Polycycl. Aromat. Compd. 2020, 1-14, https://doi.org/10.1080/10406638.2020.1823861.

17. Jilla, L.; Kolluri, P. K.; Bujji, S.; Naikal, S. Synthesis and antimicrobial agents of thiazolidinone derivatives from benzocyclohepetenone. J. Heterocycl. Chem. 2020, 57, 4078-4087, https://doi.org/10.1002/jhet.4117.

18. Patel, J. J.; Morja, M. I.; Chikhalia, K. H. An efficient synthesis of designed 4-thiazolidinone fused pyrimidine derivatives as potent antimicrobial agents. J. Heterocycl. Chem. 2020, 57, 3531-3543, https://doi.org/10.1002/jhet.4070.

19. Abu-Hashem, A. A. Synthesis and antimicrobial activity of new 1,2,4-triazole, 1,3,4-oxadiazole, 1,3,4thiadiazole, thiopyrane, thiazolidinone, and azepine derivatives. J. Heterocycl. Chem. 2021, 58, 74-92, https://doi.org/10.1002/jhet.4149.

20. Cascioferro, S.; Parrino, B.; Carbone, D.; Schillaci, D.; Giovannetti, E.; Cirrincione, G.; Diana, P. Thiazoles, their benzofused systems, and thiazolidinone derivatives: versatile and promising tools to combat antibiotic resistance. J. Med. Chem.2020, 63, 7923-7956, https://doi.org/10.1021/acs.jmedchem.9b01245.

21. Unsal-Tan, O.; Ozadali K.; Piskin K.; Balkan A. Molecular modeling, synthesis and screening of some new 4-thiazolidinone derivatives with promising selective cox-2 inhibitory activity. Eur. J. Med. Chem. 2012, 57, 59-64,https://doi.org/10.1016/j.ejmech.2012.08.046.

22. Guzel-Akdemir, O.; Carradori, S.; Grande, R.; Demir-Yazıcı, K.; Angeli, A.; Supuran, C. T.; Akdemir, A. Development of thiazolidinones as fungal carbonic anhydrase inhibitors. Int. J. Mol. Sci. 2020, 21, 2960, https://doi.org/10.3390/ijms21082960.

23. Zhang, H.; Zhang, J.; Qu, W.; Xie, S.; Huang, L.; Chen, D.; Tao, Y.; Liu, Z.; Pan, Y.; Yuan, Z. Design, synthesis, and biological evaluation of novel thiazolidinone-containing quinoxaline-1,4-di-n-oxides as antimycobacterial and antifungal agents. Front. Chem. 2020, 8, 598, https://doi.org/10.3389/fchem.2020.00598.

24. Liaras, K.; Fesatidou, M; Athina Geronikaki, A. Thiazoles and Thiazolidinones as COX/LOX inhibitors. Molecules 2018, 23, 685, https://doi.org/10.3390/molecules23030685.

25. Kulshreshtha, A.; Piplani, P. Ameliorative effects of amide derivatives of 1,3,4-thiadiazoles on scopolamine induced cognitive dysfunction. Eur. J. Med. Chem. 2016, 122, 557-573, https://doi.org/10.1016/j.ejmech.2016.06.046.

26. Su, S.; Zhou, X.; Liao, G.; Qi, P.; Jin, L. Synthesis and antibacterial evaluation of new sulfone derivatives containing 2-aroxymethyl-1,3,4-oxadiazole/thiadiazole moiety. Molecules 2016, 22, 64, https://doi.org/10.3390/molecules22010064.

27. Mishra, P. S.; Mishra, R.; Gupta, R. J.; Mazumder, R.; Malviya, R. Efficient green synthesis and biological evaluation of thiadiazole derivatives. Lett. Appl. NanoBioScience 2021, 10, 2594-2604, https://doi.org/10.33263/LIANBS103.25942604.

28. Karcz, D.; Matwijczuk, A.; Kamiński, D.; Creaven, B.; Ciszkowicz, E.; Lecka-Szlachta, K.; Starzak, K. Structural features of 1,3,4-thiadiazole-derived ligands and their $\mathrm{Zn}$ (II) and $\mathrm{Cu}(\mathrm{II})$ complexes which demonstrate synergistic antibacterial effects with kanamycin. Int. J. Mol. Sci. 2020, 21, 5735, https://doi.org/10.3390/ijms21165735.

29. Pal, S.; Singh, V.; Kumar, R.; Gogoi, R. Design and development of 1,3,4-thiadiazole based potent new nanofungicides. J. Mol. Struct. 2020, 1219, 128507, https://doi.org/10.1016/j.molstruc.2020.128507.

30. Karaburun, A.; Acar Cevik, U.; Osmaniye, D.; Saglık, B.; Kaya Çavuşoglu, B.; Levent, S.; Ozkay, Y.; Koparal, A.; Behçet, M.; Kaplancıklı, Z. Synthesis and evaluation of new 1,3,4-thiadiazole derivatives as potent antifungal agents. Molecules 2018, 23, 3129, https://doi.org/10.3390/molecules23123129. 
31. Gur, M.; Yerlikaya, S.; Sener, N.; Ozkınalı, S.; Baloglu, M. C.; Gokçe, H.; Altunoglu, Y. C.; Demir, S.; Sener, I. Antiproliferative-antimicrobial properties and structural analysis of newly synthesized schiff bases derived from some 1,3,4-thiadiazole compounds. J. Mol. Struct. 2020, 1219, 128570, https://doi.org/10.1016/j.molstruc.2020.128570.

32. Szeliga, M.; Karpinska, M.; Rola, R.; Niewiadomy, A. Design, synthesis and biological evaluation of novel 1,3,4-thiadiazole derivatives as anti-glioblastoma agents targeting the AKT pathway. Bioorganic Chem. 2020, 105, 104362, https://doi.org/10.1016/j.bioorg.2020.104362.

33. Cevik, U. A.; Osmaniye, D.; Levent, S.; Saglik, B. N.; Çavusoglu, B. K.; Ozkay, Y.; Kaplancikl, Z. A. Synthesis and characterization of a new series of thiadiazole derivatives as potential anticancer agents. Heterocycl. Commun. 2020, 26, 6-13, https://doi.org/10.1515/hc-2020-0002.

34. Muglu, H.; Sener, N.; Mohammad Emsaed, H. A.; Ozkınalı, S.; Ozkan, O. E.; Gur, M. Synthesis and characterization of 1,3,4-thiadiazole compounds derived from 4-phenoxybutyric acid for antimicrobial activities. J. Mol. Struct. 2018, 1174, 151-159, https://doi.org/10.1016/j.molstruc.2018.03.116.

35. Zabiulla; Nagesh Khadri, M. J.; Bushra Begum, A.; Sunil, M. K.; Khanum, S. A. Synthesis, docking and biological evaluation of thiadiazole and oxadiazole derivatives as antimicrobial and antioxidant agents. Results Chem. 2020, 2, 100045, https://doi.org/10.1016/j.rechem.2020.100045.

36. Taha, M.; Barak Almandil, N.; Rashid, U.; Ali, M.; Ibrahim, M.; Gollapalli, M.; Mosaddik, A.; Mohammed Khan, K. 2,5-disubstituted thiadiazoles as potent $\beta$-glucuronidase inhibitors; synthesis, in vitro and in silico studies. Bioorganic Chem. 2019, 91, 103126, https://doi.org/10.1016/j.bioorg.2019.103126.

37. El-Hazek, R. M. M.; El-Sabbagh, W. A.; El-Hazek, R. M.; El-Gazzar, M. G. Anti-inflammatory and analgesic effect of LD-RT and some novel thiadiazole derivatives through COX-2 inhibition. Arch. Pharm. 2020, 353, 2000094, https://doi.org/10.1002/ardp.202000094.

38. Saxena, V.; Sharma, C. S. Synthesis and analgesic, anti-inflammatory activities of some novel thiadiazole derivatives. International Journal of Pharmaceutical Sciences and Drug Research 2021, 13, 6.

39. Jha, A.; Murthy, Y. L. N.; Sanyal, U.; Durga, G. Rapid Synthesis, characterization, anticancer and antimicrobial activity studies of substituted thiadiazoles and their dinucleating ligand metal complexes. Med. Chem. Res. 2012, 21, 2548-2556, https://doi.org/10.1007/s00044-011-9778-y.

40. Mishra, P.; Namdeo, K.P.; Jain S.K.; Jain, S. Synthesis and antimicrobial activity of 4-thiazolidinones. Asian J. Chem. 1999, 11, 55-58.

41. Kaminskyy, D.; den Hartog, G. J. M.; Wojtyra, M.; Lelyukh, M.; Gzella, A.; Bast, A.; Lesyk, R. Antifibrotic and anticancer action of 5-ene amino/iminothiazolidinones. Eur. J. Med. Chem. 2016, 112, 180-195, https://doi.org/10.1016/j.ejmech.2016.02.011.

42. Apostolidis, I.; Liaras, K.; Geronikaki, A.; Hadjipavlou-Litina, D.; Gavalas, A.; Sokovic, M.; Glamoclija, J.; Ciric, A. Synthesis and biological evaluation of some 5-arylidene-2-(1,3-thiazol-2-ylimino)-1,3-thiazolidin4-ones as dual anti-inflammatory/antimicrobial agents. Bioorg. Med. Chem. 2013, 21, 532-539, https://doi.org/10.1016/j.bmc.2012.10.046.

43. Vogel, H.G. Drug discovery and evaluation: pharmacological assays, $3^{\text {rd }}$ ed.; Springer-Verlag Berlin Heidelberg, New York, 2008; 880-881.

44. Ellman, G. L.; Courtney, K. D.; Andres, V.; Featherstone, R. M. A new and rapid colorimetric determination of acetylcholinesterase activity. Biochem. Pharmacol. 1961, 7, 88-95, https://doi.org/10.1016/00062952(61)90145-9. 